\title{
Analysis of the Light-Curve of Comet P/Halley in 1985-1986
}

\section{David Valls-Gabaud ${ }^{1,2}$ and Angel Gómez ${ }^{1}$}

1 Ȧgrupación Astronómica de Madrid, Apartado 1039, E-28080 Madrid Spain

2 Institut d'Astrophysique de Paris 98 bis, Bld. Arago, F-75014 Paris, France

Summary. We analize the light curve of comet $\mathrm{P} /$ Halley resulting from 187 observations made by amateurs. We try to derive some constraints on the physical properties of the comet through the application of the semi-empirical photometric model (SEPM) developped by R.L. Newburn.

The $m_{1}$ (Fig. 2) and $V(1,0)$ light curves show a clear transition phase in the pre-perihelion apparition, between $\sim 1.9$ and $\sim 1.2 \mathrm{~A}$.U. which supports the division into 3 parts made by Hasegawa. It is remarkable to find that the light curve oblained by amateurs is so close to those obtained by experienced observers.

We derive an effective equivalent radius of $\beta=4.9 \pm 0.8(1 \sigma) \mathrm{km}$, and a geometrical albedo of $p_{v}=0.06 \pm 0.02(1 \sigma)$ using the method suggested by Delsemme and Rud.

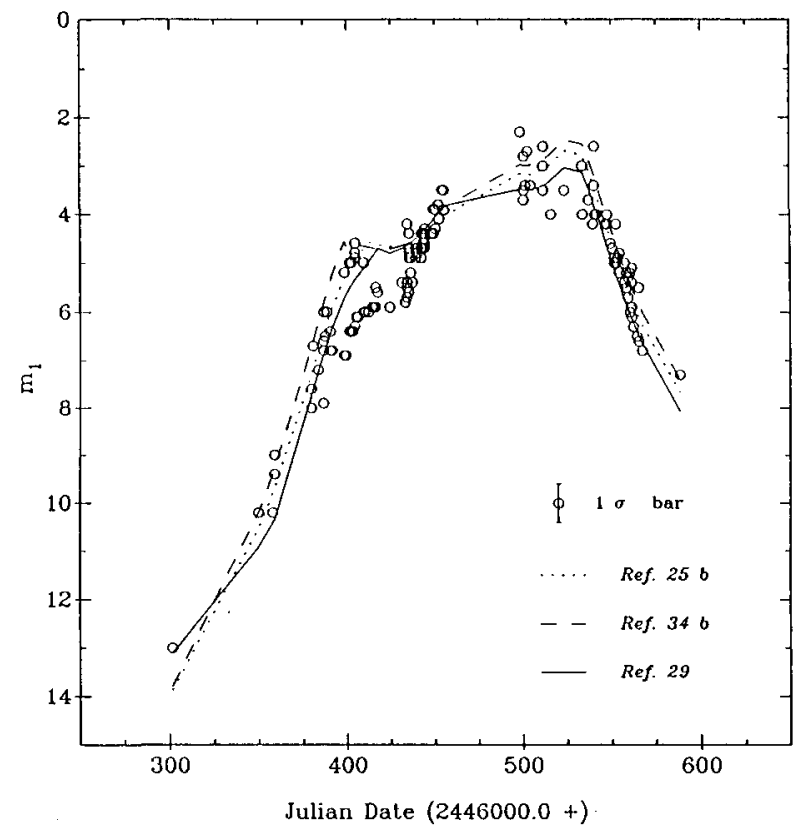

Fig. 2. A.A.M. light curve of comet $\mathrm{P} / \mathrm{Halley}$ in $1985-1986$ 


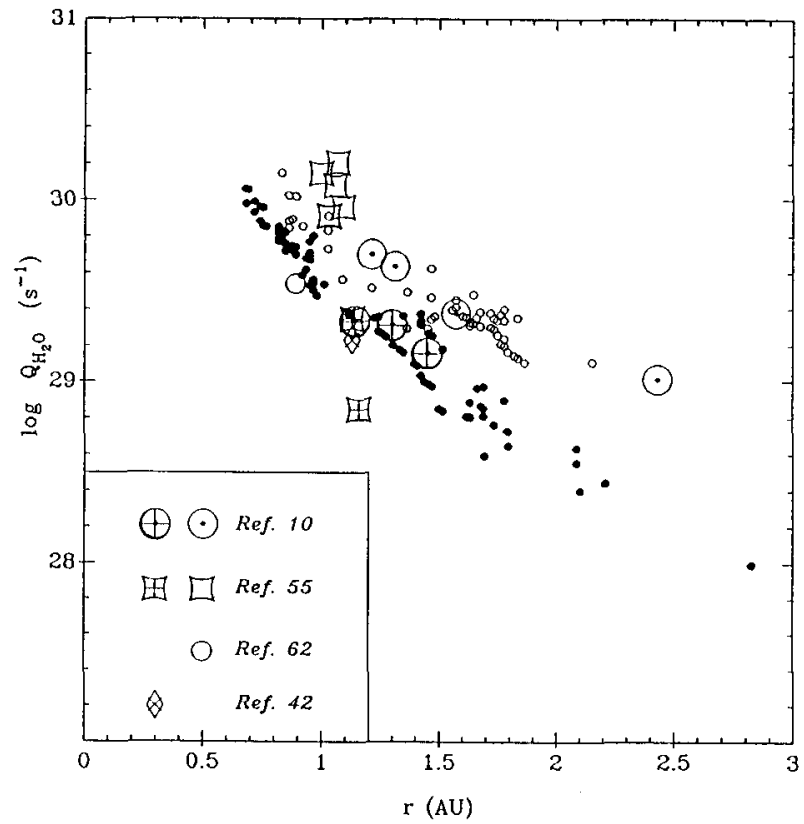

Fig. 5. Water production rate as a function of heliocentric distance. Post-perihelion (open symbols), pre-perihelion (filled symbols)

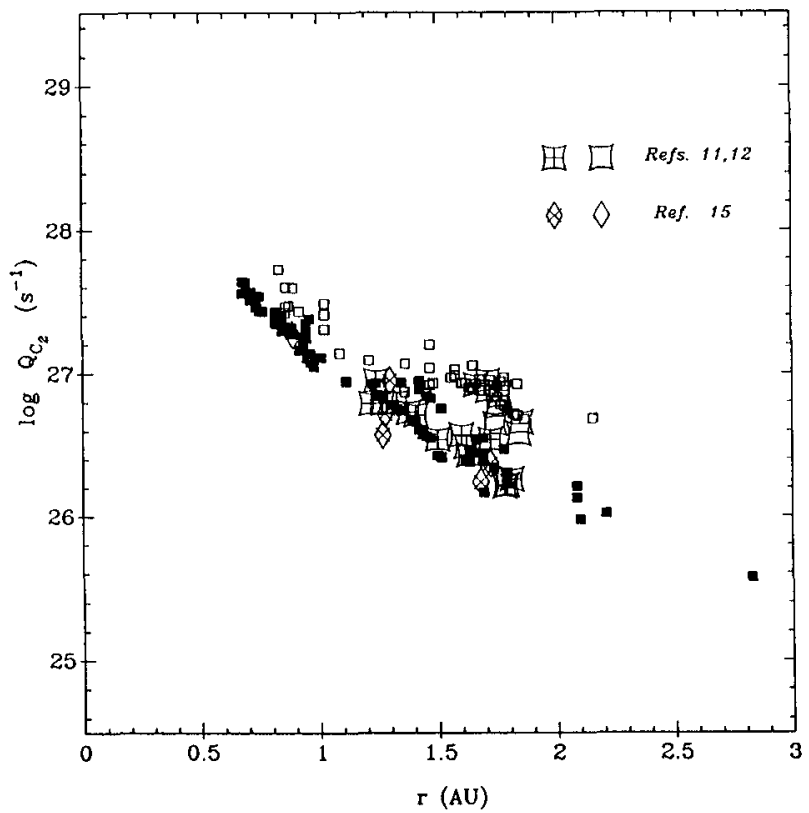

Fig. 6b. $C_{2}$ production rate, assuming a linear $(m=1)$ dependence on $Q_{H}$. Symbols as in Fig. 5 


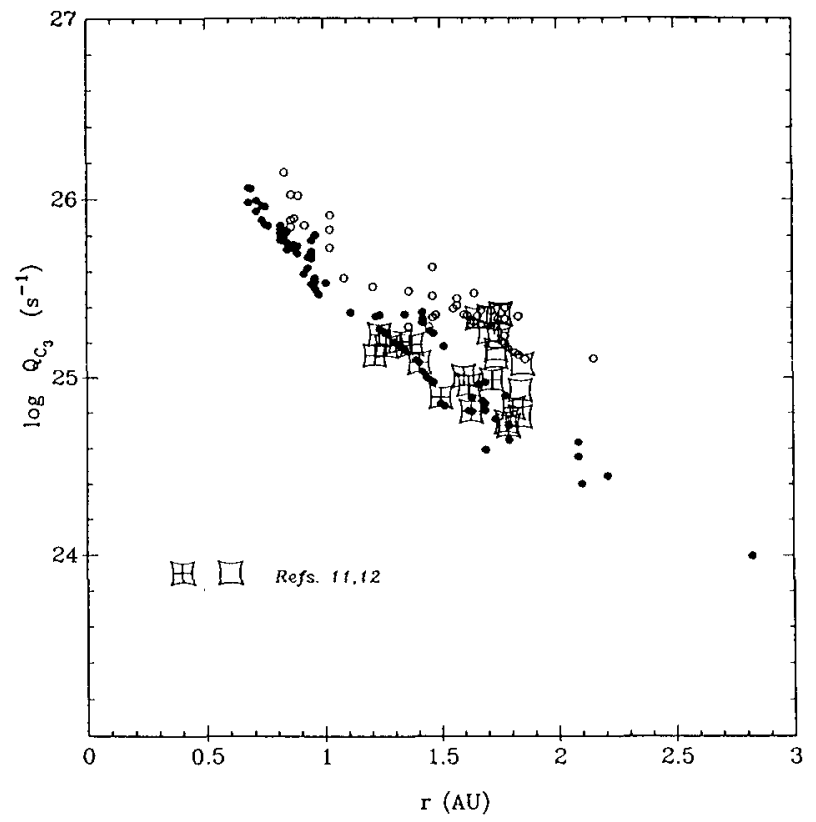

Fig. $7 C_{3}$ production rate. Symbols as in Fig. 5

The water production rate, obtained from a straightforward application of the SEPM, is illustrated in Fig. 5, where it is compared to different spectrophotometric and narrow-band photometry measures found in the literature. Note that the strongest pre-/post-perihelion asymmetry (a factor of 4-6) takes place for heliocentric distances larger than about 1.7 A.U.. This asymmetry is found in each production rate computed. A tentative explanation may be the rotation associated with a rapid change of declination of the sub-solar point, leading to the sudden sublimation of underexposed nuclear regions, as suggested by P.R. Weissman.

The $C_{2}$ production rate is shown in Fig. $6 \mathrm{~b}$, where the best results are obtained with a linear dependence on the $H$ rate, and a mixing constant of $1.510^{-32}$, consistent with the $C_{3} / C N$ ratio. The derived relative compositions are found consistent with other estimations.

We think the SEPM provides a powerful tool to find correlations to be used as constraints to the physical modelling of comets, self-consistently within factors of 2-3. This kind of analysis should be useful for amateurs that wish to interpret their own observations, provided that a detailed light curve is obtained.

A detailed account of this work has been submitted for publication to the Journal of the British Astronomical Association, and may be obtained from the authors upon request. 